\title{
Port-Hamiltonian Passivity-Based Control on SE(3) of a Fully-Actuated UAV for Aerial Physical Interaction near-Hovering
}

\author{
Ramy Rashad, Federico Califano and Stefano Stramigioli
}

\begin{abstract}
In this work, we approach the control problem of fully-actuated UAVs in a geometric port-Hamiltonian framework. The UAV is modeled as a floating rigid body on the special Euclidean group SE(3). A unified near-hovering motion and impedance controller is derived by the energy-balancing passivity-based control technique. A detailed analysis of the closed-loop system's behavior is presented for both the freeflight stability and contact stability of the UAV. The robustness of the control system to uncertainties is validated by several experiments, in which the UAV is controlled near its actuator limits. The experiments show the ability of the UAV to hover at its maximum allowed roll angle and apply its maximum allowed normal force to a surface, without the input saturation destabilizing the system.
\end{abstract}

\section{INTRODUCTION}

There is an increasing interest in the aerial robotics community to transition the use of unmanned aerial vehicles (UAVs) from passive tasks, like visual inspection and remote sensing, to active interaction tasks, like contact-based inspection and manipulation. There are two common approaches in the literature for aerial manipulation. The first approach is to endow conventional UAVs with a robotic manipulator arm [1]. However, these systems suffer from several drawbacks like inertial coupling, variable center of mass, no exertion of lateral forces, in addition to the limited payload, force exertion, and operation time.

The second common approach for aerial manipulation is the use of fully-actuated UAVs, where the UAV itself is considered as a flying end-effector which can interact with its environment. Although there have been several novel fully-actuated UAV designs introduced in the literature, most of them were introduced as a solution to the underactuation problem of conventional UAVs, e.g. [2]. The research works that focused on aerial interaction using fullyactuated UAVs include [3], where an omnidirectional barshaped hexarotor with asymmetrically-aligned rotors was introduced. The same authors extended their work in [4] and introduced an omnidirectional bar-shaped octarotor. In [5], an omnidirectional hexarotor with tiltable rotors was introduced and demonstrated for wall interaction. In [6], [7], a planar hexarotor with fixed-tilt rotors was used for contact-based inspection scenarios.

This work has been funded by the cooperation program INTERREG Deutschland-Nederland as part of the SPECTORS project number 143081.

R. Rashad and F. Califano are with the Robotics and Mechatronics group, University of Twente, Enschede, The Netherlands. Email: \{r.a.m.rashadhashem, f.califano\}eutwente.nl

S. Stramigioli is with the Robotics and Mechatronics group, University of Twente, and ITMO University, Saint Petersburg, Russia. Email: s.stramigioli@utwente.nl

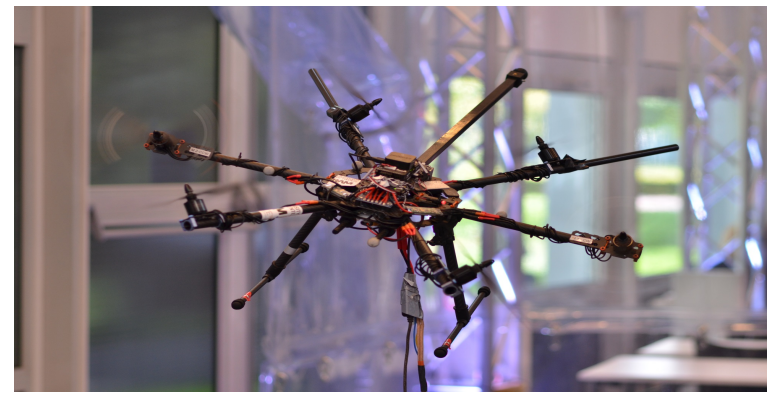

Fig. 1: Fully-actuated hexarotor hovering at a non-zero pitch and roll angle.

Several techniques have been applied in the literature for the interaction control problem. In the work of [3], [4], a hybrid pose/wrench technique was used, whereas pure motion-controllers were used for interaction in [5], [7]. The drawback of these control approaches is that they both require an accurate model of the aerial robot and the contact properties of the environment. In addition, the hybrid pose/wrench technique suffers from the problem of geometric inconsistency related to the reciprocity of wrenches and twists [8]. An admittance control approach was used for the interaction in [6], which relied on a geometric tracking controller with the rotational and translational dynamics separately considered. However, as shown in [9], a controller derived in this manner will consequently suffer from not being invariant to changes of the inertial coordinate frame.

In this work, we consider the problem of interaction control of fully-actuated UAVs in the port-Hamiltonian framework. The interaction behavior of an aerial robot can be modeled effectively and elegantly by power ports in portHamiltonian systems theory. In this paradigm the interaction is perceived as an exchange of energy between the aerial robot and the environment, instead of an independent control of pose or wrench. This is the underlying basic idea of the impedance control technique introduced in [10], and was implemented for underactuated UAVs in [11], [12].

The concepts considered in this work are presented in a geometrically consistent manner by modeling the UAV as a floating rigid body with the special Euclidean group $S E(3)$ as its configuration manifold, without separating the rotational dynamics on special orthogonal group $S O(3)$ and the translational dynamics on $\mathbb{R}^{3}$, as in [11], [12]. Our work is inspired by [13]-[15] in which the geometric impedance control problem of rigid manipulators is studied. In [13] a geometric impedance controller was designed in 


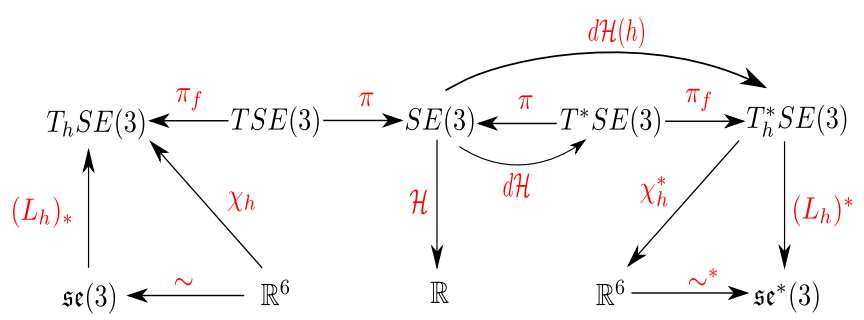

Fig. 2: Commutative Diagram relating the Lie group $S E(3)$ to the Lie algebra $\mathfrak{s e}(3)$ and its dual space $\mathfrak{s e}^{*}(3)$. The maps $\pi$ and $\pi_{f}$ represent the canonical and fiber projections, respectively.

the Lagrangian framework for a rigid manipulator, which only allowed diagonal stiffness and damping parameters. The work of [13], [14] was generalized in [15] in the portHamiltonian framework as an interconnection of mechanical structures, for robot grasping. The impedance controller of [15] enabled achieving arbitrary stiffness control and was invariant to changes of the inertial coordinate frame.

The novelty of this paper is the reformulation of the geometric impedance controller of [15] as an energybalancing passivity-based control (EB-PBC) problem for a fully-actuated UAV modeled geometrically on $S E(3)$. We extend the work of [15] by applying it for both the motion and interaction control of a UAV, in addition to a rigorous mathematical derivation and analysis of the closed loop system. Moreover, we validate the presented controller experimentally on a fully-actuated planar hexarotor to show that its passivity-based nature allows full exploitation of the UAV's capabilities. This is demonstrated by controlling the hexarotor near its control input limits.

In our recent work [16], the impedance controller, derived in this article using EB-PBC, was used in parallel with an observer-based wrench regulator to set the desired interaction wrench. The main focus of [16] was to restore the system's passivity using energy-tanks. The main contributions of this article with respect to [16] include the systematic design procedure to derive the motion and interaction controller, in addition to detailed analysis and experimental validation of the system's free-flight behavior.

\section{Mathematical Preliminaries}

The motion of objects is described in this work by the Lie group approach. The reader is referred to [17] for an introduction of the topic in robotics. Let $\left\{\Psi_{I}\right.$ : $\left.o_{I}, \hat{\boldsymbol{x}}_{I}, \hat{\boldsymbol{y}}_{I}, \hat{\boldsymbol{z}}_{I}\right\}$ denote an orthonormal inertial frame and $\left\{\Psi_{B}: o_{B}, \hat{\boldsymbol{x}}_{B}, \hat{\boldsymbol{y}}_{B}, \hat{\boldsymbol{z}}_{B}\right\}$ denote a body-fixed frame, as shown in Fig. 3. The pose of the UAV is represented by the homogeneous matrix $\boldsymbol{H}_{B}^{I}$, which is an element of the matrix Lie group $S E(3)^{1}$.

The instantaneous relative motion of the UAV $\dot{\boldsymbol{H}}_{B}^{I}$ is an element of the tangent space $T_{\boldsymbol{H}_{B}^{I}} S E(3)$, which can be mapped to an element (called twist) of the Lie algebra $\mathfrak{s e}(3)$ of the Lie group $S E(3)$. As shown in the commutative

${ }^{1} S E(3)$ is, properly speaking, the group of motions [18]. diagram in Fig 2, the map from a twist $\tilde{\boldsymbol{T}}_{B}^{B, I} \in \mathfrak{s e}(3)$ to $\dot{\boldsymbol{H}}_{B}^{I} \in T_{\boldsymbol{H}_{B}^{I}} S E(3)$ is performed by using the pushforward of the left translation map, at the identity, $\left(L_{\boldsymbol{H}_{B}^{I}}\right)_{*}$ such that

$$
\left(L_{\boldsymbol{H}_{B}^{I}}\right)_{*}\left(\tilde{\boldsymbol{T}}_{B}^{B, I}\right)=\boldsymbol{H}_{B}^{I} \tilde{\boldsymbol{T}}_{B}^{B, I}=\dot{\boldsymbol{H}}_{B}^{I} .
$$

The twist $\tilde{\boldsymbol{T}}_{B}^{B, I}$ describes the motion of $\Psi_{B}$ with respect to $\Psi_{I}$ expressed in $\Psi_{B}$, and is given by

$$
\tilde{\boldsymbol{T}}_{B}^{B, I}:=\left(\begin{array}{cc}
\tilde{\boldsymbol{\omega}}_{B}^{B, I} & \boldsymbol{v}_{B}^{B, I} \\
\mathbf{0} & 0
\end{array}\right) \in \mathfrak{s e}(3),
$$

where $\boldsymbol{v}_{B}^{B, I}$ is the UAV's linear velocity, and $\tilde{\boldsymbol{\omega}}_{B}^{B, I}:=$ $\left(\boldsymbol{R}_{B}^{I}\right)^{\top} \dot{\boldsymbol{R}}_{B}^{I}$ is an element of the Lie algebra $\mathfrak{s o}(3)$ of the Lie group $S O(3)$, with $\boldsymbol{R}_{B}^{I} \in S O(3)$ denoting the orientation of $\Psi_{B}$ with respect to $\Psi_{I}$.

The Lie algebra $\mathfrak{s o}(3)$ can be identified with $\mathbb{R}^{3}$ through the Lie algebra isomorphism

$$
\begin{aligned}
\sim: \mathbb{R}^{3} \rightarrow \mathfrak{s o}(3) \\
\boldsymbol{\omega}:=\left(\begin{array}{c}
\omega_{1} \\
\omega_{2} \\
\omega_{3}
\end{array}\right) \mapsto\left(\begin{array}{ccc}
0 & -\omega_{3} & \omega_{2} \\
\omega_{3} & 0 & -\omega_{1} \\
-\omega_{2} & \omega_{1} & 0
\end{array}\right):=\tilde{\boldsymbol{\omega}} .
\end{aligned}
$$

This allows us to represent the vector product $\wedge$ in $\mathbb{R}^{3}$ as $\tilde{\boldsymbol{\omega}} \boldsymbol{x}=\boldsymbol{\omega} \wedge \boldsymbol{x}, \forall \boldsymbol{\omega}, \boldsymbol{x} \in \mathbb{R}^{3}$. Similarly, the Lie algebra $\mathfrak{s e}(3)$ can be identified with $\mathbb{R}^{6}$ through the vector space isomorphism

$$
\begin{gathered}
\sim: \mathbb{R}^{6} \rightarrow \mathfrak{s e}(3) \\
\boldsymbol{T}:=\left(\begin{array}{c}
\boldsymbol{\omega} \\
\boldsymbol{v}
\end{array}\right) \mapsto\left(\begin{array}{ll}
\tilde{\boldsymbol{\omega}} & \boldsymbol{v} \\
\mathbf{0} & \mathbf{0}
\end{array}\right):=\tilde{\boldsymbol{T}},
\end{gathered}
$$

where, with an abuse of notation, we denote both isomorphisms by $\sim$, as it will be clear from the context. Moreover, we will call both $\boldsymbol{T}$ and $\tilde{\boldsymbol{T}}$ a twist for simplicity.

Since the Lie algebra $\mathfrak{s e}(3)$ is a vector space, its dual vector space, denoted by $\mathfrak{s e}^{*}(3)$, represents the space of wrenches (generalized forces). A wrench applied to the UAV's body expressed in $\Psi_{I}$ is denoted by $\tilde{\boldsymbol{W}}_{B}^{I}$. Moreover, the dual" map of (2) can be used to identify each element $\tilde{\boldsymbol{W}} \in \mathfrak{s e}^{*}(3)$ with an element $\boldsymbol{W}=\left(\boldsymbol{\tau}^{\top}, \boldsymbol{f}^{\top}\right)^{\top} \in \mathbb{R}^{6}$ where $\boldsymbol{f}, \boldsymbol{\tau} \in \mathbb{R}^{3}$ represent the force and torque components, respectively. Similar to the case of twists, we will call both $\boldsymbol{W}$ and $\tilde{\boldsymbol{W}}$ a wrench.

As shown in Fig. 2, using the pullback of the left translation map $\left(L_{\boldsymbol{H}_{B}^{I}}\right)^{*}$, we can map any covector $\overline{\boldsymbol{W}} \in$ $T_{\boldsymbol{H}_{B}^{I}}^{*} S E(3)$ to a wrench $\tilde{\boldsymbol{W}} \in \mathfrak{s e}^{*}(3)$. This map is defined such that

$$
\left\langle\left(L_{\boldsymbol{H}_{B}^{I}}\right)^{*}(\overline{\boldsymbol{W}}) \mid \tilde{\boldsymbol{T}}\right\rangle:=\langle\overline{\boldsymbol{W}} \mid \dot{\boldsymbol{H}}\rangle,
$$

where $\langle\cdot \mid \cdot\rangle$ denotes the vector-space dual product. The dual product on $\mathfrak{s e}(3)$ can be related to the usual dual product on $\mathbb{R}^{6}$ through (2) such that

$$
\langle\tilde{\boldsymbol{W}} \mid \tilde{\boldsymbol{T}}\rangle=\boldsymbol{W}^{\top} \boldsymbol{T}=\boldsymbol{\tau}^{\top} \boldsymbol{\omega}+\boldsymbol{f}^{\top} \boldsymbol{v}
$$

The change of coordinates of twists and wrenches between any two frames $\Psi_{j}$ and $\Psi_{k}$ is performed by

$$
\boldsymbol{T}_{\bullet}^{k, \star}=\boldsymbol{A d}_{\boldsymbol{H}_{j}^{k}} \boldsymbol{T}_{\bullet}^{j, \star}, \quad \boldsymbol{W}_{\bullet}^{j}=\boldsymbol{A} \boldsymbol{d}_{\boldsymbol{H}_{j}^{k}}^{\top} \boldsymbol{W}_{\bullet}^{k},
$$




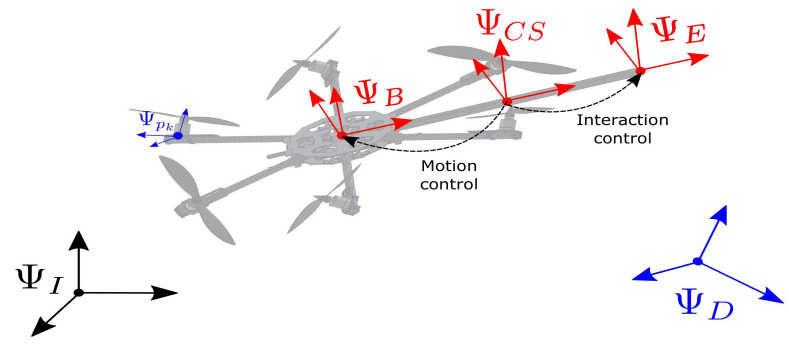

Fig. 3: Schematic view of the reference frames used.

where the map $\boldsymbol{A d}_{\boldsymbol{H}}: \mathbb{R}^{6} \rightarrow \mathbb{R}^{6}$ is the adjoint map of $\boldsymbol{H} \in S E(3)$, given by

$$
\boldsymbol{A d}_{\boldsymbol{H}}:=\left(\begin{array}{cc}
\boldsymbol{R} & \mathbf{0} \\
\tilde{\boldsymbol{\xi}} \boldsymbol{R} & \boldsymbol{R}
\end{array}\right), \quad \boldsymbol{H}:=\left(\begin{array}{cc}
\boldsymbol{R} & \boldsymbol{\xi} \\
\mathbf{0} & 1
\end{array}\right),
$$

where $\boldsymbol{\xi} \in \mathbb{R}^{3}$ denotes the displacement vector.

Finally, the following identities will be used throughout the paper. Consider the twist $\boldsymbol{T}_{j}^{j, k}$, matrices $\boldsymbol{A}, \boldsymbol{B} \in \mathbb{R}^{3 \times 3}$, $\boldsymbol{R} \in S O(3)$, and vectors $\boldsymbol{v}, \boldsymbol{w} \in \mathbb{R}^{3}$. Then the following hold:

$$
\begin{aligned}
\boldsymbol{T}_{j}^{j, k} & =-\boldsymbol{T}_{k}^{j, j}, \\
\operatorname{tr}(\tilde{\boldsymbol{v}} \tilde{\boldsymbol{w}}) & =-2 \boldsymbol{v}^{\top} \boldsymbol{w}, \\
\tilde{\boldsymbol{v}} \boldsymbol{w} & =-\tilde{\boldsymbol{w}} \boldsymbol{v}=\tilde{\boldsymbol{w}}^{\top} \boldsymbol{v}, \\
(\boldsymbol{R} \boldsymbol{v})^{\sim} & =\boldsymbol{R} \tilde{\boldsymbol{v}} \boldsymbol{R}^{\top}, \\
\operatorname{tr}(\boldsymbol{A} \boldsymbol{B}) & =\operatorname{tr}(\operatorname{sym}(\boldsymbol{A}) \operatorname{sym}(\boldsymbol{B}))+\operatorname{tr}(\operatorname{sk}(\boldsymbol{A}) \operatorname{sk}(\boldsymbol{B})), \\
\boldsymbol{v} & =\left(\operatorname{tr}(\boldsymbol{A}) \boldsymbol{I}_{3}-\boldsymbol{A}^{\top}\right) \boldsymbol{w} \Leftrightarrow \tilde{\boldsymbol{v}}=2 \operatorname{sk}(A \tilde{\boldsymbol{w}}),
\end{aligned}
$$

where $\operatorname{tr}(\cdot)$ denotes the matrix trace, while $\operatorname{sym}(\cdot)$ and $\operatorname{sk}(\cdot)$ denote the symmetric and anti-symmetric parts of a matrix, respectively.

\section{UAV DESCRIPTION}

In this work, similar to [6], [7], we consider a hexarotor UAV with fixed-tilt rotor as a case study, chosen due to its mechanical simplicity compared to other designs. To modify a traditional hexarotor in order to be fully-actuated, the six rotors should point in different directions. Each rotor's orientation is fixed and parametrized by a cant angle. Next, we derive the aerodynamic wrench applied by the six rotors to the UAV's body, followed by the choice of cant angles.

\section{A. Static Wrench Analysis}

The collectively applied wrench, which represents the control input to move the body in space, is produced by six rotors attached to the UAV's body. Let $\left\{\Psi_{p_{k}}: o_{p_{k}}, \hat{\boldsymbol{x}}_{p_{k}}, \hat{\boldsymbol{y}}_{p_{k}}, \hat{\boldsymbol{z}}_{p_{k}}\right\}$ denote the frame associated with the $k$-th propeller, where $\hat{z}_{p_{k}}$ is the direction of generated thrust, and the origin $o_{p_{k}}$ coincides with the CoM of the $k$-th propeller. We denote the displacement vector of the $k$-th propeller in $\Psi_{B}$ by

$$
\boldsymbol{\xi}_{k}:=\boldsymbol{o}_{p_{k}}^{B}=\boldsymbol{R}_{z}\left(\psi_{k}\right)[L, 0,0]^{\top},
$$

where $\boldsymbol{R}_{z}(\cdot) \in S O(3)$ is a rotation matrix about $\hat{\boldsymbol{z}}_{B}, L$ is the distance from the hexarotor's central axis $\hat{z}_{B}$ to each rotor, and the angle $\psi_{k}:=(k-1) \frac{\pi}{3}$. Moreover, we denote the thrust generation direction of the $k$-th propeller by $\boldsymbol{u}_{k}:=$ $\hat{z}_{p_{k}}=\boldsymbol{R}_{p_{k}}^{B} \hat{e}_{3}$, where $\hat{\boldsymbol{e}}_{j}$ is a vector of zeros with one at the $j$-th element, and $\boldsymbol{R}_{p_{k}}^{B}$ denotes the orientation of $\Psi_{p_{k}}$ with respect to $\Psi_{B}$ given by

$$
\boldsymbol{R}_{p_{k}}^{B}=\boldsymbol{R}_{z}\left(\psi_{k}\right) \boldsymbol{R}_{x}\left(\alpha_{k}\right),
$$

where $\alpha_{k}$ denotes the cant angles respectively.

The control wrench produced by the rotors can be derived based on a static wrench analysis. It is well known from literature that the aerodynamic thrust and drag torque of a single propeller are both proportional to the square of the propeller's spinning velocity in quasi-static flights. The thrust magnitude generated by the $k$-th propeller in $\Psi_{p_{k}}$ will be denoted by $\lambda_{k}$, while the drag torque will be expressed as $\tau_{d, k}=\gamma \sigma_{k} \lambda_{k}$, where $\gamma$ is the propeller's drag-to-thrust ratio, and $\sigma_{k} \in\{-1,1\}$ specifies the direction of the propeller's rotation. The wrench generated from each propeller on the UAV's body is expressed in $\Psi_{B}$ as

$$
\boldsymbol{W}_{p_{k}}^{B}=\lambda_{k} \boldsymbol{A d}_{\boldsymbol{H}_{B}^{p_{k}}}^{\top}\left(0,0, \gamma \sigma_{k}, 0,0,1\right)^{\top} .
$$

By summing over $k$, the cumulative aerodynamic control wrench $\boldsymbol{W}_{\mathrm{c}}^{B}$ expressed in $\Psi_{B}$ can be written as

$$
\boldsymbol{W}_{\mathrm{c}}^{B}=\left(\begin{array}{c}
\boldsymbol{\tau}_{c}^{B} \\
\boldsymbol{f}_{c}^{B}
\end{array}\right)=\sum_{k} \lambda_{k}\left(\begin{array}{c}
\tilde{\boldsymbol{\xi}}_{k} \boldsymbol{u}_{k}+\gamma \sigma_{k} \boldsymbol{u}_{k} \\
\boldsymbol{u}_{k}
\end{array}\right)=: \boldsymbol{M} \boldsymbol{\lambda},
$$

where $\boldsymbol{\lambda}=\left[\lambda_{1}, \cdots, \lambda_{6}\right]^{\top}$, and $\boldsymbol{M} \in \mathbb{R}^{6 \times 6}$ is the control allocation matrix.

\section{B. Choice of the cant angles}

The fully-actuated hexarotor considered in this work is optimized following the work of [7]. The objective of the optimization process is to maximize the aerodynamic wrench that can be generated by the propellers on the body of the UAV. This in turn will maximize the agility of the UAV, the maximum angle the UAV can hover at, and the interaction wrench that the UAV can apply to the environment in physical contact.

The optimized ${ }^{2}$ cant-angles, for the choice of objective, are $\alpha_{k}=(-1)^{k+1} \alpha^{*}$, where $\alpha^{*}=47^{\circ}$, while the rotation directions of the rotors are given by $\sigma_{k}=(-1)^{k}$.

\section{UAV PORT-HAMILTONIAN MODELING}

Now we present the port-Hamiltonian dynamic model of the UAV. The state of the system comprises of the UAV's pose $\boldsymbol{H}_{B}^{I}$ and its generalized momentum given by $\boldsymbol{P}^{B}:=$ $\mathcal{I} \boldsymbol{T}_{B}^{B, I}$, where $\mathcal{I} \in \mathbb{R}^{6 \times 6}$ denotes the generalized inertia tensor expressed in $\Psi_{B}$. Thus, the state is given by

$$
\overline{\boldsymbol{x}}=\left(\boldsymbol{H}_{B}^{I}, \boldsymbol{P}^{B}\right) \in S E(3) \times \mathbb{R}^{6} .
$$

The Hamiltonian of the system is given by the sum of the UAV's kinetic and potential energies:

$$
\begin{aligned}
\mathcal{H}(\overline{\boldsymbol{x}}) & =\mathcal{H}_{\mathrm{kin}}\left(\boldsymbol{P}^{B}\right)+\mathcal{H}_{\mathrm{grv}}\left(\boldsymbol{H}_{B}^{I}\right) \\
& :=\frac{1}{2}\left(\boldsymbol{P}^{B}\right)^{\top} \mathcal{I}^{-1} \boldsymbol{P}^{B}+m\left(\boldsymbol{\xi}_{B}^{I}\right)^{\top} \boldsymbol{g},
\end{aligned}
$$

\footnotetext{
${ }^{2}$ The details of the optimization process are omitted as the optimizationbased design is out of the scope of this paper.
} 
where $m$ is the UAV's mass, $\boldsymbol{g}:=g \hat{\boldsymbol{z}}_{I}$ is the gravity vector (inverse), and $g$ is the gravitational acceleration constant.

The UAV's dynamic model in the port-Hamiltonian framework [19], is given by

$$
\begin{aligned}
\frac{d}{d t}\left(\begin{array}{c}
\boldsymbol{H}_{B}^{I} \\
\boldsymbol{P}^{B}
\end{array}\right) & =\left(\begin{array}{cc}
\mathbf{0} & \chi_{\boldsymbol{H}_{B}^{I}} \\
-\chi_{\boldsymbol{H}_{B}^{I}}^{*} & \left(\boldsymbol{P}^{B}\right)^{\times}
\end{array}\right)\left(\begin{array}{l}
\partial \mathcal{H} / \partial \boldsymbol{H}_{B}^{I} \\
\partial \mathcal{H} / \partial \boldsymbol{P}^{B}
\end{array}\right)+\left(\begin{array}{c}
\mathbf{0} \\
\boldsymbol{I}_{6}
\end{array}\right) \boldsymbol{W}^{B}, \\
\boldsymbol{T}_{B}^{B, I} & =\left(\begin{array}{ll}
\mathbf{0} & \boldsymbol{I}_{6}
\end{array}\right)\left(\begin{array}{l}
\partial \mathcal{H} / \partial \boldsymbol{H}_{B}^{I} \\
\partial \mathcal{H} / \partial \boldsymbol{P}^{B}
\end{array}\right)
\end{aligned}
$$

where the map $\chi$ and its dual $\chi^{*}$ are the compositions defined in Fig 2. The skew-symmetric matrix $\left(\boldsymbol{P}^{B}\right)^{\times}$is given by

$$
(\boldsymbol{P})^{\times}:=\left(\begin{array}{cc}
\tilde{\boldsymbol{P}}_{\omega} & \tilde{\boldsymbol{P}}_{v} \\
\tilde{\boldsymbol{P}}_{v} & \mathbf{0}
\end{array}\right), \quad \boldsymbol{P}=\left(\begin{array}{c}
\boldsymbol{P}_{\omega} \\
\boldsymbol{P}_{v}
\end{array}\right) \in \mathbb{R}^{6} .
$$

Finally, The total wrench applied to the UAV's rigid body is given by

$$
\boldsymbol{W}^{B}=\boldsymbol{W}_{\mathrm{c}}^{B}+\boldsymbol{W}_{\mathrm{int}}^{B},
$$

with $\boldsymbol{W}_{\mathrm{c}}^{B}$ denoting the aerodynamic control wrench (12), and $\boldsymbol{W}_{\text {int }}^{B}$ denoting the interaction wrench applied to the UAV by the environment.

\section{ENERGY-BALANCING PASSIVITy-BASED CONTROL}

Now we present the stabilization control system design of the port-Hamiltonian system (15) for both motion and interaction control. The control approach used is the EnergyBalancing Passivity-Based Control (EB-PBC) approach [20]. The formulation of the UAV geometric control design as an EB-PBC problem is one of the contributions of this work.

\section{A. Change of Coordinates}

With reference to Fig. 3, let $\Psi_{D}$ denote the (virtual) desired frame of the UAV. For simplicity of presentation, it is useful to introduce another body-fixed frame, called the center of stiffness frame $\Psi_{C S}$, which has the same orientation as $\Psi_{B}$ but a different location. The location of $\Psi_{C S}$ will vary according to the control task ${ }^{3}$. For motion control $\Psi_{C S}$ coincides $\Psi_{B}$ (i.e. $\boldsymbol{\xi}_{C S}^{B}=0$ ), while for interaction control $\Psi_{C S}$ coincides with the end-effector frame $\Psi_{E}$.

Now we introduce a change of coordinates of the state (13) such that the new system's state $\boldsymbol{x}$ is given by

$$
\boldsymbol{x}=\Phi(\overline{\boldsymbol{x}}):=\left(\boldsymbol{H}_{I}^{D} \boldsymbol{H}_{B}^{I} \boldsymbol{H}_{C S}^{B}, \boldsymbol{P}^{B}\right)=\left(\boldsymbol{H}_{C S}^{D}, \boldsymbol{P}^{B}\right) .
$$

The time derivative of $\boldsymbol{H}_{C S}^{D}$ can be written in the new coordinates as

$$
\begin{aligned}
\dot{\boldsymbol{H}}_{C S}^{D} & =\chi_{\boldsymbol{H}_{C S}^{D}}\left(\boldsymbol{T}_{C S}^{C S, D}\right)=\chi_{\boldsymbol{H}_{C S}^{D}}\left(\boldsymbol{A} \boldsymbol{d}_{\boldsymbol{H}_{B}^{C S}} \boldsymbol{T}_{C S}^{B, D}\right) \\
& =\chi_{\boldsymbol{H}_{C S}^{D}}\left(\boldsymbol{A} \boldsymbol{d}_{\boldsymbol{H}_{B}^{C S}} \boldsymbol{T}_{B}^{B, I}\right),
\end{aligned}
$$

where (5) was used. The equality of $\boldsymbol{T}_{C S}^{B, D}$ and $\boldsymbol{T}_{B}^{B, I}$ is due to the fact that the twist of $\Psi_{B}$ is the same as $\Psi_{C S}$, in addition to the assumption that $\boldsymbol{T}_{D}^{D, I}=0$ for regulation control.

\footnotetext{
${ }^{3}$ In general, the $\Psi_{C S}$ frame can be made variable [21].
}

By rewriting the time derivative of $\boldsymbol{P}^{B}$ in the new coordinates similar to the aforementioned argument, the open-loop port-Hamiltonian system (15) can be rewritten now as

$$
\begin{aligned}
& \dot{\boldsymbol{x}}=\boldsymbol{J}(\boldsymbol{x}) \frac{\partial \mathcal{H}(\boldsymbol{x})}{\partial \boldsymbol{x}}+\boldsymbol{G} \boldsymbol{W}^{B}, \\
& \boldsymbol{y}=\boldsymbol{G}^{\top} \frac{\partial \mathcal{H}(\boldsymbol{x})}{\partial \boldsymbol{x}},
\end{aligned}
$$

where $\boldsymbol{W}^{B}$ is given by (16), $\boldsymbol{y}=\boldsymbol{T}_{B}^{B, I}$ is the output, $\boldsymbol{G}^{\top}=\left(\begin{array}{ll}\mathbf{0} & \boldsymbol{I}_{6}\end{array}\right)$ is the input matrix, and the skew-symmetric interconnection operator $\boldsymbol{J}(\boldsymbol{x})$ is given by

$$
\boldsymbol{J}(\boldsymbol{x})=\left(\begin{array}{cc}
\mathbf{0} & \chi_{\boldsymbol{H}_{C S}^{D} \circ \boldsymbol{A} \boldsymbol{d}_{\boldsymbol{H}_{B}^{C S}}}{\left(\boldsymbol{P}^{B}\right)^{\times}}_{-\boldsymbol{A} \boldsymbol{d}_{\boldsymbol{H}_{B}^{\top C S}}^{\top} \circ \chi_{\boldsymbol{H}_{C S}^{D}}^{*}}
\end{array}\right),
$$

with $\circ$ denoting the composition operator.

\section{B. Control Objective}

Considering the port Hamiltonian system (17), the control objective (in the absence of $\boldsymbol{W}_{\text {int }}^{B}$ ) is to find a control law

$$
\boldsymbol{W}_{\mathrm{c}}^{B}=\boldsymbol{W}_{\mathrm{es}}^{B}(\boldsymbol{x})+\boldsymbol{W}_{\mathrm{di}}^{B},
$$

such that the closed loop system will retain the portHamiltonian structure:

$$
\begin{aligned}
& \dot{\boldsymbol{x}}=\boldsymbol{J}(\boldsymbol{x}) \frac{\partial \mathcal{H}_{\mathrm{cl}}(\boldsymbol{x})}{\partial \boldsymbol{x}}+\boldsymbol{G} \boldsymbol{W}_{\mathrm{di}}^{B}, \\
& \boldsymbol{y}=\boldsymbol{G}^{\top} \frac{\partial \mathcal{H}_{\mathrm{cl}}(\boldsymbol{x})}{\partial \boldsymbol{x}},
\end{aligned}
$$

with the closed-loop Hamiltonian $\mathcal{H}_{\mathrm{cl}}$ presenting a strict minimum at $\boldsymbol{x}^{*}=\left(\boldsymbol{I}_{4}, \mathbf{0}\right)$. The first term of the control law $\boldsymbol{W}_{\mathrm{es}}^{B}$ is the state feedback component responsible for the energy shaping, while $\boldsymbol{W}_{\mathrm{di}}^{B}$ is the output feedback component responsible for injecting damping such that asymptotic stabilization is achieved.

\section{Matching Equation}

For the UAV's model (17), in which internal dissipation is not present, the closed loop energy $\mathcal{H}_{\mathrm{cl}}$ is chosen as

$$
\mathcal{H}_{\mathrm{cl}}(\boldsymbol{x})=\mathcal{H}(\boldsymbol{x})+\mathcal{H}_{\mathrm{a}}(\boldsymbol{x}),
$$

where $\mathcal{H}_{\mathrm{a}}$ is the energy added by the controller to the system. By substituting (18) in (17), and (20) in (19), then comparing both systems together, we get the matching equation

$$
\boldsymbol{J}(\boldsymbol{x}) \frac{\partial \mathcal{H}_{\mathrm{a}}(\boldsymbol{x})}{\partial \boldsymbol{x}}=\boldsymbol{G} \boldsymbol{W}_{\mathrm{es}}^{B}(\boldsymbol{x}),
$$

which should be solved to find the energy shaping control term $\boldsymbol{W}_{\mathrm{es}}^{B}$. It can be shown that the matching equation reduces to

$$
\begin{aligned}
\partial \mathcal{H}_{\mathrm{a}} / \partial \boldsymbol{P}^{B} & =0, \\
-\boldsymbol{A} \boldsymbol{d}_{\boldsymbol{H}_{B}^{C S}}^{\top} \circ \chi_{\boldsymbol{H}_{C S}^{D}}^{*}\left(\partial \mathcal{H}_{\mathrm{a}} / \partial \boldsymbol{H}_{C S}^{D}\right) & =\boldsymbol{W}_{\mathrm{es}}^{B} .
\end{aligned}
$$

The solution of (22) implies that $\mathcal{H}_{\mathrm{a}}(\boldsymbol{x})=\mathcal{H}_{\mathrm{a}}\left(\boldsymbol{H}_{C S}^{D}\right)$. Now the control problem reduces to solving (23) for a choice of $\mathcal{H}_{\mathrm{a}}$, which will be discussed next. 


\section{Energy Shaping Control Procedure}

From the definition of $\chi_{\boldsymbol{H}_{C S}^{D}}^{*}$, we can expand (23) as

$$
\begin{aligned}
\boldsymbol{W}_{\mathrm{es}}^{B} & =\boldsymbol{A} \boldsymbol{d}_{\boldsymbol{H}_{B}^{C S}}^{\top} \boldsymbol{W}_{\mathrm{es}}^{C S}, \\
\tilde{\boldsymbol{W}}_{\mathrm{es}}^{C S} & =-\left(L_{\boldsymbol{H}_{C S}^{D}}\right)^{*}\left(d \mathcal{H}_{\mathrm{a}}\left(\boldsymbol{H}_{C S}^{D}\right)\right),
\end{aligned}
$$

where the covector $d \mathcal{H}_{\mathrm{a}}\left(\boldsymbol{H}_{C S}^{D}\right) \in T_{\boldsymbol{H}_{C S}^{D}}^{*} S E(3)$ denotes the differential of the smooth function $\mathcal{H}_{\mathrm{a}}: S E(3) \rightarrow \mathbb{R}$ at $\boldsymbol{H}_{C S}^{D}$. Similar to (3), the action of the pullback in (25) is defined as

$$
\left\langle\tilde{\boldsymbol{W}}_{\mathrm{es}}^{C S} \mid \delta \tilde{\boldsymbol{T}}_{D}^{C S}\right\rangle=\left\langle d \mathcal{H}_{\mathrm{a}}\left(\boldsymbol{H}_{C S}^{D}\right) \mid d \boldsymbol{H}_{C S}^{D}\right\rangle,
$$

with $d \boldsymbol{H}_{C S}^{D_{S}}$ expressed, by using (6), as

$$
d \boldsymbol{H}_{C S}^{D}=\boldsymbol{H}_{C S}^{D} \delta \tilde{\boldsymbol{T}}_{C S}^{C S, D}=-\left(L_{\boldsymbol{H}_{C S}^{D}}\right)_{*}\left(\delta \tilde{\boldsymbol{T}}_{D}^{C S}\right),
$$

with $\delta \tilde{\boldsymbol{T}}_{D}^{C S}$ as a shorthand notation for $\delta \tilde{\boldsymbol{T}}_{D}^{C S, C S} \in \mathfrak{s e}(3)$ which is an infinitesimal twist displacement.

Remark 1: Note that the pairing in (26) represents infinitesimal virtual work, in contrast to the pairing in (3) which represents mechanical power, which is the first-order time-derivative of the energy. Similar to [14], we choose to follow the variational approach for the controller derivation.

The right-hand side of (26) can be calculated by

$$
\left\langle d \mathcal{H}_{\mathrm{a}}\left(\boldsymbol{H}_{C S}^{D}\right) \mid d \boldsymbol{H}_{C S}^{D}\right\rangle=\mathcal{H}_{\mathrm{a}}\left(\boldsymbol{H}_{C S}^{D}+d \boldsymbol{H}_{C S}^{D}\right)-\mathcal{H}_{\mathrm{a}}\left(\boldsymbol{H}_{C S}^{D}\right) .
$$

From (4), we can express the left-hand side of (26) as

$$
\left\langle\tilde{\boldsymbol{W}}_{\mathrm{es}}^{C S} \mid \delta \tilde{\boldsymbol{T}}_{D}^{C S}\right\rangle=\left(\boldsymbol{\tau}_{\mathrm{es}}^{C S}\right)^{\top} \delta \boldsymbol{\theta}_{D}^{C S}+\left(\boldsymbol{f}_{\mathrm{es}}^{C S}\right)^{\top} \delta \boldsymbol{\xi}_{D}^{C S},
$$

where $\delta \boldsymbol{\theta}_{D}^{C S}, \delta \boldsymbol{\xi}_{D}^{C S} \in \mathbb{R}^{3}$ are components of $\delta \boldsymbol{T}_{D}^{C S} \in \mathbb{R}^{6}$, related to the components of $d \boldsymbol{H}_{C S}^{D}$ by

$$
d \boldsymbol{R}_{C S}^{D}=-\boldsymbol{R}_{C S}^{D} \delta \tilde{\boldsymbol{\theta}}_{D}^{C S}, \quad d \boldsymbol{\xi}_{C S}^{D}=-\boldsymbol{R}_{C S}^{D} \delta \boldsymbol{\xi}_{D}^{C S} .
$$

Using identity (7), it will be useful to express (29) as

$$
\left\langle\tilde{\boldsymbol{W}}_{\mathrm{es}}^{C S} \mid \delta \tilde{\boldsymbol{T}}_{D}^{C S}\right\rangle=-\frac{1}{2} \operatorname{tr}\left(\tilde{\boldsymbol{\tau}}_{\mathrm{es}}^{C S} \delta \tilde{\boldsymbol{\theta}}_{D}^{C S}\right)-\frac{1}{2} \operatorname{tr}\left(\tilde{\boldsymbol{f}}_{\mathrm{es}}^{C S} \delta \tilde{\boldsymbol{\xi}}_{D}^{C S}\right) .
$$

\section{E. EB-PBC Control Law}

In this work, the controller's added energy is chosen as

$$
\mathcal{H}_{\mathrm{a}}\left(\boldsymbol{H}_{C S}^{D}\right)=\mathcal{H}_{\mathrm{p}}\left(\boldsymbol{H}_{C S}^{D}\right)-\mathcal{H}_{\mathrm{grv}}\left(\boldsymbol{H}_{C S}^{D}\right)
$$

where $\mathcal{H}_{\text {grv }}$ is given by

$$
\mathcal{H}_{\mathrm{grv}}\left(\boldsymbol{H}_{C S}^{D}\right)=m\left(\boldsymbol{\xi}_{D}^{I}+\boldsymbol{R}_{D}^{I} \boldsymbol{\xi}_{C S}^{D}+\boldsymbol{R}_{D}^{I} \boldsymbol{R}_{C S}^{D} \boldsymbol{\xi}_{B}^{C S}\right)^{\top} \boldsymbol{g},
$$

and $\mathcal{H}_{\mathrm{p}}$ is the elastic potential energy [15] given by

$$
\begin{aligned}
& \mathcal{H}_{\mathrm{p}}(\boldsymbol{R}, \boldsymbol{\xi})=\mathcal{H}_{\mathrm{t}, 1}(\boldsymbol{\xi})+\mathcal{H}_{\mathrm{t}, 2}(\boldsymbol{R}, \boldsymbol{\xi})+\mathcal{H}_{\mathrm{o}}(\boldsymbol{R}) \\
& :=\frac{1}{4} \boldsymbol{\xi}^{\top} \boldsymbol{K}_{t} \boldsymbol{\xi}+\frac{1}{4} \boldsymbol{\xi}^{\top} \boldsymbol{R} \boldsymbol{K}_{t} \boldsymbol{R}^{\top} \boldsymbol{\xi}-\operatorname{tr}\left(\boldsymbol{G}_{o}\left(\boldsymbol{R}-\boldsymbol{I}_{3}\right)\right) .
\end{aligned}
$$

The potential energy function (33) is parameterized by the translational stiffness matrix $\boldsymbol{K}_{t} \in \mathbb{R}^{3 \times 3}$ and the orientational co-stiffness matrix $\boldsymbol{G}_{o} \in \mathbb{R}^{3 \times 3}$. Stiffness and costiffness matrices are related to each other by

$$
\boldsymbol{K}_{x}=\operatorname{tr}\left(\boldsymbol{G}_{x}\right) \boldsymbol{I}_{3}-\boldsymbol{G}_{x}, \quad \boldsymbol{G}_{x}=\frac{1}{2} \operatorname{tr}\left(\boldsymbol{K}_{x}\right) \boldsymbol{I}_{3}-\boldsymbol{K}_{x},
$$

for $x \in\{t, o\}$. Both $\boldsymbol{K}_{x}, \boldsymbol{G}_{x}$ are symmetric and their eigenvectors coincide with one another i.e.

$$
\boldsymbol{K}_{x}=\boldsymbol{R}_{x} \Lambda_{x} \boldsymbol{R}_{x}^{\top}, \quad \boldsymbol{G}_{x}=\boldsymbol{R}_{x} \Gamma_{x} \boldsymbol{R}_{x}^{\top},
$$

where $\boldsymbol{R}_{x}=\left(\boldsymbol{e}_{x_{1}}, \boldsymbol{e}_{x_{2}}, \boldsymbol{e}_{x_{3}}\right) \in S O(3)$ represents the principal axes of stiffness, while $\Gamma_{x}=\operatorname{diag}\left(\gamma_{x_{1}}, \gamma_{x_{2}}, \gamma_{x_{3}}\right)$ and $\Lambda_{x}=\operatorname{diag}\left(\lambda_{x_{1}}, \lambda_{x_{2}}, \lambda_{x_{3}}\right)$ represent the principal stiffness and co-stiffness values along the axes, respectively.

Assumption 1: For $i=\{1,2,3\}$, the principal stiffness gains $\gamma_{x_{i}}$ are strictly positive and chosen such that the principal costiffness gains $\lambda_{x_{i}}$ are distinct.

Remark 2: Note that in (33), the first term is the usual potential energy considered for a translational spring, while the third term represents a misalignment of $\boldsymbol{R}$ from the identity [9]. The second term in (33) is the term that allows for arbitrary non-diagonal stiffness $\boldsymbol{K}_{t}$ such that the controller maintains invariance to changes of the inertial coordinate frame $\Psi_{I}$ [9], [15]. This term can be only included by considering the dynamics fully on $S E(3)$ without splitting the rotational and translational dynamics.

For the choice of the added energy (32), the EB-PBC control law $\boldsymbol{W}_{\mathrm{c}}^{B}$ is given by

$$
\begin{aligned}
\boldsymbol{W}_{\mathrm{c}}^{B} & =\boldsymbol{A} \boldsymbol{d}_{\boldsymbol{H}_{B}^{C} S}^{\top}\left(\boldsymbol{W}_{\mathrm{p}}^{C S}-\boldsymbol{W}_{\mathrm{grv}}^{C S}\right)+\boldsymbol{W}_{\mathrm{di}}^{B}, \\
\tilde{\boldsymbol{\tau}}_{\mathrm{p}}^{C S} & =-2 \operatorname{sk}\left(\boldsymbol{G}_{o} \boldsymbol{R}_{C S}^{D}\right)-\operatorname{sk}\left(\boldsymbol{G}_{t} \boldsymbol{R}_{D}^{C S} \tilde{\boldsymbol{\xi}}_{C S}^{D} \tilde{\boldsymbol{\xi}}_{C S}^{D} \boldsymbol{R}_{C S}^{D}\right), \\
\tilde{\boldsymbol{f}}_{\mathrm{p}}^{C S} & =-\boldsymbol{R}_{D}^{C S} \operatorname{sk}\left(\boldsymbol{G}_{t} \tilde{\boldsymbol{\xi}}_{C S}^{D}\right) \boldsymbol{R}_{C S}^{D}-\operatorname{sk}\left(\boldsymbol{G}_{t} \boldsymbol{R}_{D}^{C S} \tilde{\boldsymbol{\xi}}_{C S}^{D} \boldsymbol{R}_{C S}^{D}\right), \\
\boldsymbol{\tau}_{\mathrm{grv}}^{C S} & =-m \tilde{\boldsymbol{\xi}}_{B}^{C S} \boldsymbol{R}_{D}^{C S} \boldsymbol{R}_{I}^{D} \boldsymbol{g}, \quad \boldsymbol{f}_{\mathrm{grv}}^{C S}=-m \boldsymbol{R}_{D}^{C S} \boldsymbol{R}_{I}^{D} \boldsymbol{g}, \\
\boldsymbol{W}_{\mathrm{di}}^{B} & =-\boldsymbol{K}_{d} \boldsymbol{T}_{B}^{B, I},
\end{aligned}
$$

where $\boldsymbol{K}_{d} \in \mathbb{R}^{6 \times 6}$ is a symmetric positive definite matrix.

\section{F. Control Law Derivation}

Now, we present the derivation of the energy-shaping control law (37-39) for each separate energy term in (32).

1) Orientational energy: For the orientational energy $\mathcal{H}_{\mathrm{o}}\left(\boldsymbol{R}_{C S}^{D}\right)$,from the definition of $\mathcal{H}_{\mathrm{o}}$ and (28), we get

$$
\begin{aligned}
\left\langle d \mathcal{H}_{\mathrm{o}}\left(\boldsymbol{R}_{C S}^{D}\right) \mid d \boldsymbol{R}_{C S}^{D}\right\rangle= & -\operatorname{tr}\left(\boldsymbol{G}_{o}\left(\boldsymbol{R}_{C S}^{D}+d \boldsymbol{R}_{C S}^{D}-\boldsymbol{I}_{3}\right)\right) \\
& +\operatorname{tr}\left(\boldsymbol{G}_{o}\left(\boldsymbol{R}_{C S}^{D}-\boldsymbol{I}_{3}\right)\right), \\
= & -\operatorname{tr}\left(\boldsymbol{G}_{o} d \boldsymbol{R}_{C S}^{D}\right), \\
= & \operatorname{tr}\left(\boldsymbol{G}_{o} \boldsymbol{R}_{C S}^{D} \delta \tilde{\boldsymbol{\theta}}_{D}^{C S}\right), \\
= & \operatorname{tr}\left(\operatorname{sk}\left(\boldsymbol{G}_{o} \boldsymbol{R}_{C S}^{D}\right) \delta \tilde{\boldsymbol{\theta}}_{D}^{C S}\right),
\end{aligned}
$$

where the second equality results from the linearity of the trace map, the third equality results from (30), and the last equality results from identity (10).

By comparing equations (41) and (31), we conclude that the corresponding control wrench for $\mathcal{H}_{\mathrm{o}}\left(\boldsymbol{R}_{C S}^{D}\right)$ is given by

$$
\tilde{\boldsymbol{\tau}}_{\mathrm{o}}^{C S}=-2 \operatorname{sk}\left(\boldsymbol{G}_{o} \boldsymbol{R}_{C S}^{D}\right), \quad \tilde{\boldsymbol{f}}_{\mathrm{o}}^{C S}=\mathbf{0} .
$$

2) Translational energy: By following the same steps for the first term of the translational energy $\mathcal{H}_{\mathrm{t}, 1}(\boldsymbol{\xi})$, we get

$$
\begin{gathered}
\left\langle d \mathcal{H}_{\mathrm{t}, 1}\left(\boldsymbol{\xi}_{C S}^{D}\right) \mid d \boldsymbol{\xi}_{C S}^{D}\right\rangle=\frac{1}{4}\left(\boldsymbol{R}_{C S}^{D} \delta \boldsymbol{\xi}_{D}^{C S}\right)^{\top} \boldsymbol{K}_{t}\left(\boldsymbol{R}_{C S}^{D} \delta \boldsymbol{\xi}_{D}^{C S}\right) \\
-\frac{1}{4}\left(\boldsymbol{R}_{C S}^{D} \delta \boldsymbol{\xi}_{D}^{C S}\right)^{\top} \boldsymbol{K}_{t} \boldsymbol{\xi}_{C S}^{D}-\frac{1}{4}\left(\boldsymbol{\xi}_{C S}^{D}\right)^{\top} \boldsymbol{K}_{t} \boldsymbol{R}_{C S}^{D} \delta \boldsymbol{\xi}_{D}^{C S}
\end{gathered}
$$


where the first can be neglected since it is a second order term [14], thus we get

$$
\left\langle d \mathcal{H}_{\mathrm{t}, 1}\left(\boldsymbol{\xi}_{C S}^{D}\right) \mid d \boldsymbol{\xi}_{C S}^{D}\right\rangle=-\frac{1}{2}\left(\delta \boldsymbol{\xi}_{D}^{C S}\right)^{\top}\left(\boldsymbol{R}_{C S}^{D}\right)^{\top} \boldsymbol{K}_{t} \boldsymbol{\xi}_{C S}^{D} .
$$

By comparison to (29), we conclude that

$$
\boldsymbol{f}_{\mathrm{t}, 1}^{C S}=-\frac{1}{2} \boldsymbol{R}_{D}^{C S} \boldsymbol{K}_{t} \boldsymbol{\xi}_{C S}^{D}, \quad \boldsymbol{\tau}_{\mathrm{t}, 1}^{C S}=\mathbf{0}
$$

By using (34) and the identity (11), we can show that $\left(\boldsymbol{K}_{t} \boldsymbol{\xi}_{C S}^{D}\right)^{\sim}=2 \operatorname{sk}\left(\boldsymbol{G}_{t} \tilde{\boldsymbol{\xi}}_{C S}^{D}\right)$, which allows us to rewrite (43) as

$$
\tilde{\boldsymbol{f}}_{\mathrm{t}, 1}^{C S}=-\boldsymbol{R}_{D}^{C S} \operatorname{sk}\left(\boldsymbol{G}_{t} \tilde{\boldsymbol{\xi}}_{C S}^{D}\right) \boldsymbol{R}_{C S}^{D}, \quad \tilde{\boldsymbol{\tau}}_{\mathrm{t}, 1}^{C S}=\mathbf{0},
$$

where the identity (9) was used. To simplify the control derivation for the second term of the translational energy $\mathcal{H}_{\mathrm{t}, 2}(\boldsymbol{\xi})$, it is useful [15] to rewrite it as

$\mathcal{H}_{\mathrm{t}, 2}\left(\boldsymbol{H}_{C S}^{D}\right)=\mathcal{H}_{\mathrm{t}, 2}\left(\boldsymbol{R}_{C S}^{D}, \boldsymbol{\xi}_{C S}^{D}\right)=-\frac{1}{4} \operatorname{tr}\left(\tilde{\boldsymbol{\xi}}_{C S}^{D} \boldsymbol{R}_{C S}^{D} \boldsymbol{G}_{t} \boldsymbol{R}_{D}^{C S} \tilde{\boldsymbol{\xi}}_{C S}^{D}\right)$

By following the same line of thought and some lengthy calculations [15], we can express the infinitesimal work as

$$
\begin{aligned}
\left\langle d \mathcal{H}_{\mathrm{t}, 2}\left(\boldsymbol{H}_{C S}^{D}\right) \mid d \boldsymbol{H}_{C S}^{D}\right\rangle & =\frac{1}{2} \operatorname{tr}\left(\operatorname{sk}\left(\boldsymbol{G}_{t} \boldsymbol{R}_{D}^{C S} \tilde{\boldsymbol{\xi}}_{C S}^{D} \tilde{\boldsymbol{\xi}}_{C S}^{D} \boldsymbol{R}_{C S}^{D}\right) \delta \tilde{\boldsymbol{\theta}}_{D}^{C S}\right) \\
& +\frac{1}{2} \operatorname{tr}\left(\operatorname{sk}\left(\boldsymbol{G}_{t} \boldsymbol{R}_{D}^{C S} \tilde{\boldsymbol{\xi}}_{C S}^{D} \boldsymbol{R}_{C S}^{D}\right) \delta \tilde{\boldsymbol{\xi}}_{D}^{C S}\right) .
\end{aligned}
$$

By comparison to (31), we conclude that

$$
\begin{aligned}
& \tilde{\boldsymbol{f}}_{\mathrm{t}, 2}^{C S}=-\operatorname{sk}\left(\boldsymbol{G}_{t} \boldsymbol{R}_{D}^{C S} \tilde{\boldsymbol{\xi}}_{C S}^{D} \boldsymbol{R}_{C S}^{D}\right), \\
& \tilde{\boldsymbol{\tau}}_{\mathrm{t}, 2}^{C S}=-\operatorname{sk}\left(\boldsymbol{G}_{t} \boldsymbol{R}_{D}^{C S} \tilde{\boldsymbol{\xi}}_{C S}^{D} \tilde{\boldsymbol{\xi}}_{C S}^{D} \boldsymbol{R}_{C S}^{D}\right) .
\end{aligned}
$$

By using $(8,11,34)$, we can rewrite the force component as

$$
\boldsymbol{f}_{\mathrm{t}, 2}^{C S}=-\frac{1}{2} \boldsymbol{K}_{t} \boldsymbol{R}_{D}^{C S} \boldsymbol{\xi}_{C S}^{D} .
$$

3) Gravity compensation: Finally, by evaluating the infinitesimal work (28) for the gravitational energy $\mathcal{H}_{\text {grv }}\left(\boldsymbol{H}_{C S}^{D}\right)$ we get

$$
\begin{aligned}
& \left\langle d \mathcal{H}_{\mathrm{grv}}\left(\boldsymbol{\xi}_{C S}^{D}\right) \mid d \boldsymbol{\xi}_{C S}^{D}\right\rangle \\
& \quad=-m\left[\left(\boldsymbol{R}_{D}^{I} \boldsymbol{R}_{C S}^{D} \delta \boldsymbol{\xi}_{D}^{C S}\right)^{\top}+\left(\boldsymbol{R}_{D}^{I} \boldsymbol{R}_{C S}^{D} \delta \tilde{\boldsymbol{\theta}}_{D}^{C S} \boldsymbol{\xi}_{C S}^{B}\right)^{\top}\right] \boldsymbol{g} \\
& \quad=-m\left(\delta \boldsymbol{\xi}_{D}^{C S}\right)^{\top} \boldsymbol{R}_{D}^{C S} \boldsymbol{R}_{I}^{D} \boldsymbol{g}-m\left(\delta \boldsymbol{\theta}_{D}^{C S}\right)^{\top} \tilde{\boldsymbol{\xi}}_{B}^{C S} \boldsymbol{R}_{D}^{C S} \boldsymbol{R}_{I}^{D} \boldsymbol{g}
\end{aligned}
$$

where identity (8) was used. By comparison to (29), we get

$$
\boldsymbol{f}_{\mathrm{grv}}^{C S}=-m \boldsymbol{R}_{D}^{C S} \boldsymbol{R}_{I}^{D} \boldsymbol{g}, \quad \boldsymbol{\tau}_{\mathrm{grv}}^{C S}=\tilde{\boldsymbol{\xi}}_{B}^{C S} \boldsymbol{f}_{\mathrm{grv}}^{C S} .
$$

This concludes the control law derivation.

\section{Closed Loop System Behavior}

In this section, we examine the closed loop system's behavior. Using the control law (36) we can write the closed loop system as

$$
\dot{\boldsymbol{x}}=(\boldsymbol{J}(\boldsymbol{x})-\boldsymbol{R}) \frac{\partial \mathcal{H}_{\mathrm{cl}}(\boldsymbol{x})}{\partial \boldsymbol{x}}+\boldsymbol{G} \boldsymbol{W}_{\mathrm{int}}^{B},
$$

with the damping matrix $\boldsymbol{R}$ given by

$$
\boldsymbol{R}=\left(\begin{array}{cc}
\mathbf{0} & \mathbf{0} \\
\mathbf{0} & \boldsymbol{K}_{d}
\end{array}\right)
$$

and the closed loop Hamiltonian given by $\mathcal{H}_{\mathrm{cl}}=\mathcal{H}_{\mathrm{kin}}+$ $\mathcal{H}_{\mathrm{t}, 1}+\mathcal{H}_{\mathrm{t}, 2}+\mathcal{H}_{\mathrm{o}}$. In what follows, we present the almost ${ }^{4}$ global asymptotic stability of the system in free flight $\left(\boldsymbol{W}_{\text {int }}^{B}=\mathbf{0}, \boldsymbol{H}_{C S}^{B}=\boldsymbol{I}_{4}\right)$, followed by the contact stability and impedance behavior of the system in contact $\left(\boldsymbol{W}_{\text {int }}^{B} \neq\right.$ $\left.\mathbf{0}, \boldsymbol{H}_{C S}^{B}=\boldsymbol{H}_{E}^{B}\right)$

\section{A. Free-flight Stability}

The boundedness of $\mathcal{H}_{\mathrm{cl}}$ can be proven by the fact that its first three terms are quadratic in $\boldsymbol{P}^{B}, \boldsymbol{\xi}_{C S}^{D}$, and $\left(\boldsymbol{R}_{D}^{C S} \boldsymbol{\xi}_{C S}^{D}\right)$ respectively, thus are bounded from below. As for $\mathcal{H}_{\mathrm{o}}$, due to its smoothness and the compactness of $S O(3)$ as a topological space, $\mathcal{H}_{\mathrm{o}}$ is also bounded from below [22].

As a consequence of assumption 1 , the closed loop system has four isolated equilibrium points $\boldsymbol{x}_{i}^{*}=\left(\boldsymbol{H}_{i}^{*}, \boldsymbol{P}_{i}^{*}\right)$, where for all four points $\boldsymbol{\xi}_{i}^{*}=\mathbf{0}$ and $\boldsymbol{P}_{i}^{*}=\mathbf{0}$. The equilibrium orientations are given by [22]

$$
\boldsymbol{R}_{i}^{*}=\exp \left(\pi \tilde{\boldsymbol{e}}_{o_{i}}\right), \quad i=\{1,2,3\}, \quad \boldsymbol{R}_{4}^{*}=\boldsymbol{I}_{3},
$$

where $e_{o_{i}}$ are the principal axes of $\boldsymbol{K}_{o}$, as shown in (35).

The time derivative of $\mathcal{H}_{\mathrm{cl}}$ is given by

$$
\begin{aligned}
\dot{\mathcal{H}}_{\mathrm{cl}} & =\frac{\partial \mathcal{H}_{\mathrm{cl}}{ }^{\top}}{\partial \boldsymbol{x}}(\boldsymbol{J}-\boldsymbol{R}) \frac{\partial \mathcal{H}_{\mathrm{cl}}}{\partial \boldsymbol{x}}, \\
& =-\left(\boldsymbol{P}^{B}\right)^{\top} \mathcal{I}^{-\top} \boldsymbol{K}_{d} \mathcal{I}^{-1} \boldsymbol{P}^{B} \leq 0,
\end{aligned}
$$

which follows from the skew-symmetry of $\boldsymbol{J}$, and the positive definiteness of $\boldsymbol{K}_{d}$ and $\mathcal{I}$. Thus, we conclude the system's passivity using $\mathcal{H}_{\mathrm{cl}}$ as a storage function.

By applying LaSalle's invariance principle, it can be shown that the largest invariance set consists of the union of all equilibrium points $\boldsymbol{x}_{i}^{*}$, which results from

$$
\dot{\mathcal{H}}_{\mathrm{cl}}=0 \Rightarrow \boldsymbol{P}^{B}=\mathbf{0} \Rightarrow \dot{\boldsymbol{P}}^{B}=\mathbf{0} \Rightarrow \boldsymbol{W}_{\mathrm{p}}^{C S}\left(\boldsymbol{H}_{C S}^{D}\right)=0 .
$$

Thus, all solutions of the system (48), converge to one of the four points. However, it will turn out that only the equilibrium $\boldsymbol{x}_{4}^{*}=\left(\boldsymbol{I}_{4}, \mathbf{0}\right)$ is asymptotically stable, while the rest are unstable. This can be shown by examining the local linearized behavior of the control term $\boldsymbol{W}_{\mathrm{p}}^{C S}\left(\boldsymbol{H}_{C S}^{D}\right)$ around the equilibrium points. By substituting

$$
\boldsymbol{R}_{C S}^{D}=\boldsymbol{R}_{i}^{*}-\boldsymbol{R}_{i}^{*} \delta \tilde{\boldsymbol{\theta}}_{D}^{C S}, \quad \boldsymbol{\xi}_{C S}^{D}=\mathbf{0}-\boldsymbol{R}_{i}^{*} \delta \boldsymbol{\xi}_{D}^{C S},
$$

in equations $(42,43,45,46)$, and discarding high order terms, we get

$$
\begin{aligned}
\tilde{\boldsymbol{\tau}}_{\mathrm{o}}^{C S} & =2 \operatorname{sk}\left(\boldsymbol{G}_{o} \boldsymbol{R}_{i}^{*} \delta \tilde{\boldsymbol{\theta}}_{D}^{C S}\right), & \tilde{\boldsymbol{\tau}}_{\mathrm{t}, 2}^{C S} & =\mathbf{0}, \\
\boldsymbol{f}_{\mathrm{t}, 1}^{C S} & =\frac{1}{2}\left(\boldsymbol{R}_{i}^{*}\right)^{\top} \boldsymbol{K}_{t} \boldsymbol{R}_{i}^{*} \delta \boldsymbol{\xi}_{D}^{C S}, & \boldsymbol{f}_{\mathrm{t}, 2}^{C S} & =\frac{1}{2} \boldsymbol{K}_{t} \delta \boldsymbol{\xi}_{D}^{C S},
\end{aligned}
$$

which allows rewriting the equivalent wrench in $(37,38)$ as

$$
\boldsymbol{W}_{\mathrm{p}}^{C S}=\boldsymbol{W}_{\mathrm{p}}^{B}=-\boldsymbol{K}_{i}^{*} \delta \boldsymbol{T}_{B}^{B, I}=\boldsymbol{K}_{i}^{*} \delta \boldsymbol{T}_{D}^{C S},
$$

with $\boldsymbol{K}_{i}^{*} \in \mathbb{R}^{6 \times 6}$ given by

$$
\boldsymbol{K}_{i}^{*}=\left(\begin{array}{cc}
\operatorname{tr}\left(\boldsymbol{G}_{o} \boldsymbol{R}_{i}^{*}\right) \boldsymbol{I}_{3}-\left(\boldsymbol{G}_{o} \boldsymbol{R}_{i}^{*}\right)^{\top} & \mathbf{0} \\
\mathbf{0} & \frac{1}{2}\left[\left(\boldsymbol{R}_{i}^{*}\right)^{\top} \boldsymbol{K}_{t} \boldsymbol{R}_{i}^{*}+\boldsymbol{K}_{t}\right]
\end{array}\right) .
$$

${ }^{4}$ As is it impossible topologically to achieve global asymptotic stability on $S O(3)$ and consequently $S E(3)$. 
By evaluating the stiffness matrix $\boldsymbol{K}_{i}^{*}$ at $\boldsymbol{R}_{4}^{*}=\boldsymbol{I}_{3}$, we get the positive definite matrix

$$
\boldsymbol{K}_{s}:=\boldsymbol{K}_{4}^{*}=\left(\begin{array}{cc}
\boldsymbol{K}_{o} & \mathbf{0} \\
\mathbf{0} & \boldsymbol{K}_{t}
\end{array}\right),
$$

which shows that the potential control term (52) resembles a linear elastic wrench near the equilibrium $\boldsymbol{R}_{4}^{*}$. Similar to the analysis in [13], it can be shown that the stiffness matrix evaluated at the remaining equilibrium points is not positive definite. Finally, this concludes the almost global asymptotic stability of the equilibrium point $\boldsymbol{x}_{4}^{*}=\left(\boldsymbol{I}_{4}, \mathbf{0}\right)$.

\section{B. Interaction behavior}

In the interaction control mode, the passivity of the system with respect to the interaction power port $\left(\boldsymbol{T}_{B}^{B, I}, \boldsymbol{W}_{\text {int }}^{B}\right)$ can be analyzed by rewriting (51) as

$$
\begin{aligned}
\dot{\mathcal{H}}_{\mathrm{cl}} & =-\left(\boldsymbol{P}^{B}\right)^{\top} \mathcal{I}^{-\top} \boldsymbol{K}_{d} \mathcal{I}^{-1} \boldsymbol{P}^{B}+\left(\mathcal{I}^{-1} \boldsymbol{P}^{B}\right)^{\top} \boldsymbol{W}_{\mathrm{int}}^{B} \\
& \leq\left(\boldsymbol{W}_{\mathrm{int}}^{B}\right)^{\top} \boldsymbol{T}_{B}^{B, I} .
\end{aligned}
$$

This guarantees the contact stability of the UAV with any passive arbitrary environment.

The impedance behavior of the UAV's end effector with respect to the environment can be calculated by representing all terms of the momentum dynamics in (48) in frame $\Psi_{E}$ using (5). The linearized impedance behavior in terms of an infinitesimal twist displacement $\delta \boldsymbol{T}_{E}^{E, D}$ can be shown to be

$$
\mathcal{I}^{E} \delta \ddot{\boldsymbol{T}}_{E}^{E, D}+\boldsymbol{K}_{d}^{E} \delta \dot{\boldsymbol{T}}_{E}^{E, D}+\boldsymbol{K}_{s} \delta \boldsymbol{T}_{E}^{E, D}=\boldsymbol{W}_{\mathrm{int}}^{E},
$$

where the apparent inertia $\mathcal{I}^{E}$ and damping $\boldsymbol{K}_{d}^{E}$ matrices are given, respectively, by

$$
\mathcal{I}^{E}=\boldsymbol{A} \boldsymbol{d}_{\boldsymbol{H}_{E}^{B}}^{\top} \mathcal{I} \boldsymbol{A} \boldsymbol{d}_{\boldsymbol{H}_{E}^{B}}, \quad \boldsymbol{K}_{d}^{E}=\boldsymbol{A} \boldsymbol{d}_{\boldsymbol{H}_{E}^{B}}^{\top} \boldsymbol{K}_{d} \boldsymbol{A} \boldsymbol{d}_{\boldsymbol{H}_{E}^{B}},
$$

while the stiffness matrix $\boldsymbol{K}_{s}$ is given by (53).

To conclude, during contact with the environment, the EBPBC control law (36) acts as an impedance controller with the impedance behavior (55).

Remark 3: Note that the passivity result in (54) actually holds for any external power port to the system. Consider the actual control wrench in (16) which is equal to the desired control wrench in (36) only if $\left(\Delta \boldsymbol{W}_{c}=\mathbf{0}\right)$ which represents the wrench due to modeling uncertainties like unmodeled dynamics or input constraints. In case $\Delta \boldsymbol{W}_{c} \neq \mathbf{0}$, the closed loop system (48) would have an additional term $\left(\boldsymbol{G} \Delta \boldsymbol{W}_{c}\right)$, and the system's passivity can be easily shown as in (54).

\section{EXPERIMENTAL VALIDATION}

The experimental setup used to validate the proposed control approach consists of a fully-actuated hexarotor UAV with unidirectional rotors. Details of the UAV and software architecture used to implement the control law (36-40) can be found in [16]. The experiments are conducted in an indoor lab with a motion-capture system. The experiments consist of two scenarios, one for interaction control and the other for motion control. The controller gains in both experiments are chosen as $\boldsymbol{K}_{t}=\operatorname{diag}(12,12,12), \boldsymbol{K}_{o}=\operatorname{diag}(0.8,0.8,0.5)$, and $\boldsymbol{K}_{d}=\operatorname{diag}(3.6,3.5,2.1,7,7,7)$. The results are presented next, while recordings of the experiments can be found in the supplementary video.

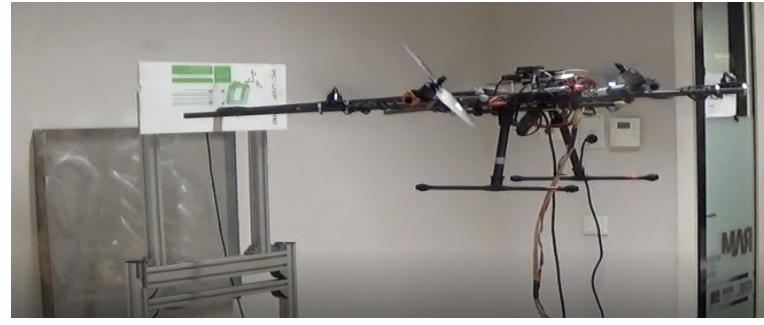

Fig. 4: Experiment 1: Snapshot $(t=215 \mathrm{~s})$ showing the UAV hovering with one rotor off and applying an $\approx 10 \mathrm{~N}$ force to a vertical surface rigidly connected to a force/torque sensor.
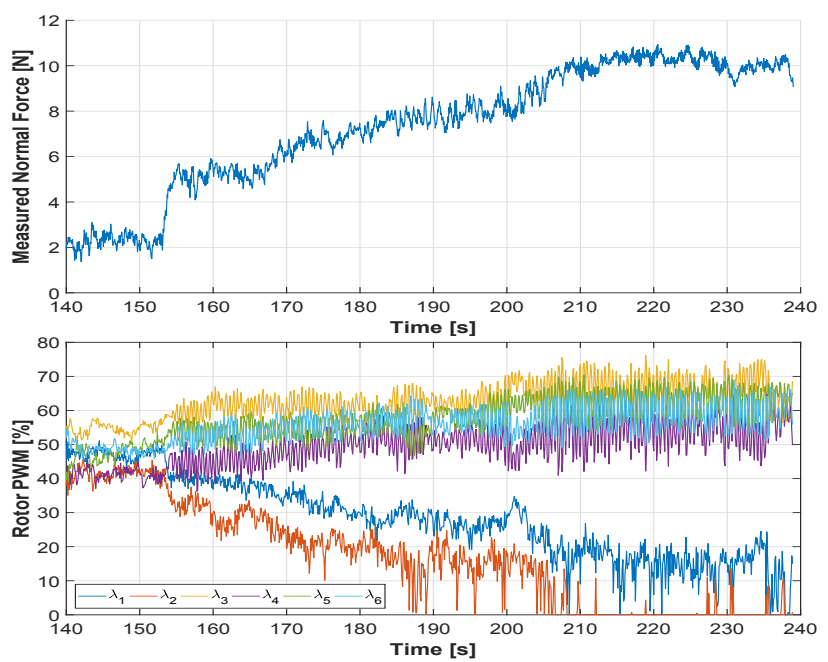

Fig. 5: Experiment 1: Measured normal force applied by the UAV to the vertical surface (top). The commanded rotor PWM signals (bottom).

\section{A. Experimental Results}

The first experiment comprises of the UAV applying a normal force to a vertical surface rigidly connected to an ATI mini40 force/torque sensor (ATI Industrial Automation), as shown in Fig 4. The measured normal force and commanded rotor PWM signals are displayed in Fig. 5. The applied force is increased by slowly increasing $\boldsymbol{\xi}_{E}^{D}$, until its maximum allowed normal force is achieved, as shown by the force measurement in the top plot. In the bottom plot, it can be seen that this maximum force is achieved with $\lambda_{2}$ at its lower limit. Therefore, there are instants in which the hexarotor interacts while hovering with its second motor turned off, as shown in the snapshot in Fig. 4.

The second experiment consists of the UAV hovering at different roll angles. In Fig. 6, snapshots of the UAV hovering at an angle of 0,15 , and $25 \mathrm{deg}$ are shown. The UAV is commanded to hover at the roll angle $\phi_{B}=25 \mathrm{deg}$ for $t \geq 130$ s. As shown in Fig. 7, for the UAV to hover at this maximum angle, $\lambda_{4}$ is commanded to be zero for an interval about 40 seconds. Thus, the UAV hovers at this maximum angle with five rotors only. However, due to the inability of the UAV to maintain its desired position against wind disturbances, the UAV drifts which then requires a non-zero $\lambda_{4}$ to be able to reduce the position error. 


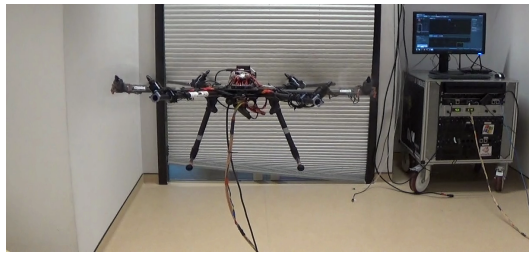

(a) $\phi_{B}=0^{\circ}, t=20 \mathrm{~s}$

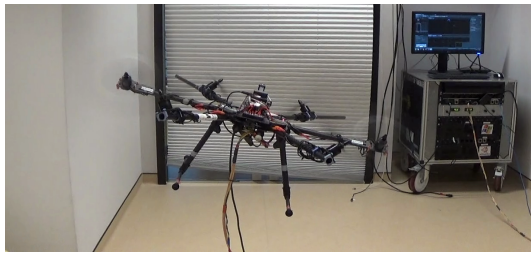

(b) $\phi_{B}=15^{\circ}, t=68 \mathrm{~s}$

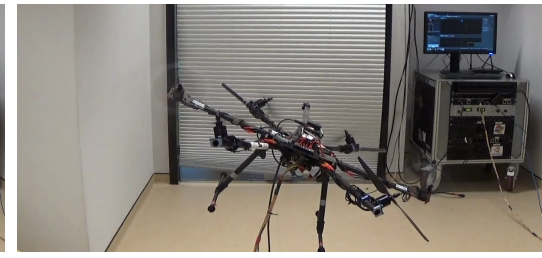

(c) $\phi_{B}=25^{\circ}, t=141 \mathrm{~s}$

Fig. 6: Experiment 2: Snapshots of the UAV hovering at different roll angles $\phi_{B}$. The right snapshot shows the UAV hovering at its maximum allowed angle with one of its rotors off.

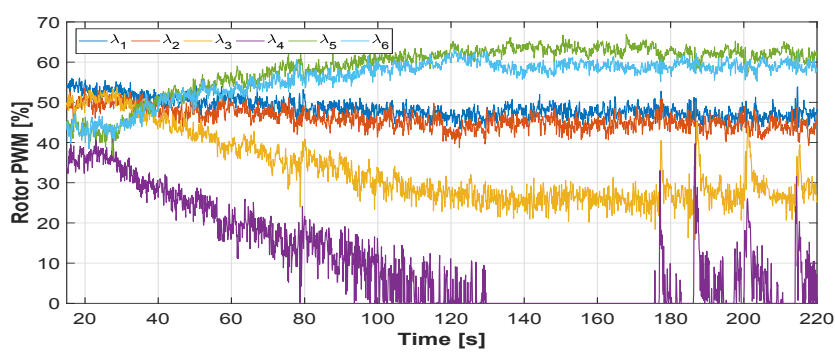

Fig. 7: Experiment 2: The commanded rotor PWM signals where the fourth rotor hits its lower limit.

The main feature of the controller that the two aforementioned experiments demonstrate is its robustness to the actuator limits and in general any uncertainties in the model as discussed in Remark 3. Therefore, near the UAV's maximum physical capabilities, the nonlinear saturation of the motor inputs did not destabilize the system.

\section{CONCLUSION}

In this work we presented a geometric port-Hamiltonian controller for a class of fully actuated UAVs. The controller was designed using the EB-PBC approach. Although the port-Hamiltonian control of fully-actuated mechanical systems is a well studied topic, the problem considered in this work was not trivial, due to the non-symplectic structure of the dynamic model. In addition, the stiffness parameters can be chosen arbitrary without losing geometric consistency as discussed in Remark 2. Moreover, the geometric notation allowed for a compact derivation and rigorous mathematical analysis of the control system. The experiments performed show the validity and robustness of the control approach especially to unmodeled uncertainties like input saturation.

\section{ACKNOWLEDGMENT}

The authors would like to thank Jelmer Goerres for his help with the UAV's design.

\section{REFERENCES}

[1] F. Ruggiero, V. Lippiello, and A. Ollero, "Aerial manipulation: A literature review," IEEE Robotics and Automation Letters, vol. 3, no. 3 , pp. 1957-1964, 2018.

[2] D. Brescianini and R. D'Andrea, "Design, modeling and control of an omni-directional aerial vehicle," in 2016 IEEE Int. Conf. Robot. Autom., pp. 3261-3266, IEEE, may 2016.

[3] S. Park, J. Her, J. Kim, and D. Lee, "Design, modeling and control of omni-directional aerial robot," in 2016 IEEE/RSJ Int. Conf. Intell. Robot. Syst., pp. 1570-1575, IEEE, oct 2016.
[4] S. Park, J. Lee, J. Ahn, M. Kim, J. Her, G.-H. Yang, and D. Lee, "Odar: Aerial manipulation platform enabling omnidirectional wrench generation," IEEE/ASME Transactions on Mechatronics, vol. 23, no. 4, pp. 1907-1918, 2018.

[5] M. Kamel, S. Verling, O. Elkhatib, C. Sprecher, P. Wulkop, Z. Taylor, R. Siegwart, and I. Gilitschenski, "The voliro omniorientational hexacopter: An agile and maneuverable tiltable-rotor aerial vehicle," IEEE Robotics \& Automation Magazine, vol. 25, no. 4, pp. 34-44, 2018.

[6] M. Ryll, G. Muscio, F. Pierri, E. Cataldi, G. Antonelli, F. Caccavale, and A. Franchi, "6D physical interaction with a fully actuated aerial robot," in 2017 IEEE International Conference on Robotics and Automation, 2017.

[7] G. Jiang, R. M. Voyles, and J. J. Choi, "Precision fully-actuated uav for visual and physical inspection of structures for nuclear decommissioning and search and rescue," in 2018 IEEE International Symposium on Safety, Security, and Rescue Robotics (SSRR), pp. 1-7, IEEE, 2018.

[8] J. Duffy, "The fallacy of modern hybrid control theory that is based on orthogonal complements of twist and wrench spaces," Journal of Robotic Systems, vol. 7, no. 2, pp. 139-144, 1990.

[9] F. Bullo and R. M. Murray, "Tracking for fully actuated mechanical systems: a geometric framework," Automatica, vol. 35, no. 1, pp. $17-$ 34, 1999.

[10] N. Hogan, "Impedance control: An approach to manipulation," in 1984 American control conference, pp. 304-313, IEEE, 1984.

[11] J. Acosta, M. Sanchez, and A. Ollero, "Robust control of underactuated Aerial Manipulators via IDA-PBC," in Decision and Control (CDC), IEEE 53rd Annual Conference on, pp. 673-678, IEEE, 2014.

[12] B. Yüksel, C. Secchi, H. H. Bülthoff, and A. Franchi, "Aerial physical interaction via ida-pbc," The International Journal of Robotics Research, p. 0278364919835605, 2019.

[13] E. D. Fasse and J. F. Broenink, "A spatial impedance controller for robotic manipulation," IEEE Transactions on Robotics and Automation, vol. 13, no. 4, pp. 546-556, 1997.

[14] E. D. Fasse, "On the spatial compliance of robotic manipulators," Journal of dynamic systems, measurement, and control, vol. 119, no. 4, pp. 839-844, 1997.

[15] S. Stramigioli, Modeling and IPC control of interactive mechanical system - A coordinate-free approach. Springer-Verlag London, 2001.

[16] R. Rashad, J. B. C. Engelen, and S. Stramigioli, "Energy tank-based wrench/impedance control of a fully-actuated hexarotor: A geometric port-hamiltonian approach," in IEEE International Conference on Robotics and Automation (ICRA), pp. 6418-6424, 2019.

[17] R. M. Murray, S. S. Sastry, and L. Zexiang, A Mathematical Introduction to Robotic Manipulation. Boca Raton, FL, USA: CRC Press, Inc., 1st ed., 1994.

[18] S. Stramigioli and H. Bruyninckx, "Non-intrinsicity of references in rigid body motions," in IEEE International Conference on Robotics and Automation, vol. 1, pp. 13-18, IEEE, 2000.

[19] A. Van Der Schaft and B. Maschke, "Port-hamiltonian systems on graphs," SIAM Journal on Control and Optimization, vol. 51, no. 2, pp. 906-937, 2013.

[20] R. Ortega, A. J. Van Der Schaft, I. Mareels, and B. Maschke, "Putting energy back in control," IEEE Control Systems, vol. 21, no. 2, pp. 1833, 2001.

[21] S. Stramigioli and V. Duindam, "Variable spatial springs for robot control," in 2001 IEEE/RSJ International Conference on Intelligent Robots and Systems, IROS 2001, pp. 1906-1911, IEEE, 2001.

[22] F. Bullo and A. D. Lewis, Geometric control of mechanical systems: modeling, analysis, and design for simple mechanical control systems, vol. 49. Springer Science \& Business Media, 2004. 